\title{
Maintaining a frozen shipping environment for Phase I clinical trial distribution
}

\author{
M.A. Elliott*, G.W. Halbert \\ Cancer Research UK Formulation Unit, Strathclyde Institute for Pharmacy and Biomedical Sciences, \\ University of Strathclyde, Glasgow, Scotland G1 1XW, United Kingdom \\ Received 24 January 2007; received in revised form 4 June 2007; accepted 6 June 2007 \\ Available online 14 June 2007
}

\begin{abstract}
The need for stringent temperature control provides significant challenges to pharmaceutical distributors operating in all sectors of the industry. Products with a frozen storage label requirement can be significantly problematic. This study aimed to provide evidence of robust and reproducible frozen shipment arrangements to be operated by a Phase I clinical trial unit. Dry ice was used to achieve a deep frozen internal parcel environment and was tested in a laboratory setting using ultra low temperature loggers within dummy product packs within the test parcels. The laboratory dry ice packing configuration was then repeatedly tested in real time transits using a Glasgow to London delivery schedule. An internal temperature specification was set to not exceed $-10^{\circ} \mathrm{C}$ during the transport. During each delivery, external temperature monitoring measured the temperature stress experienced by the box in transit. Results demonstrated the ability of the chosen system to not exceed $-13.6^{\circ} \mathrm{C}$ on average $\left(-10^{\circ} \mathrm{C}\right.$ maximum) when exposed to external temperatures of up to $+20.1^{\circ} \mathrm{C}$ (mean kinetic temperature). The effect was maintained for at least $52.5 \mathrm{~h}$.
\end{abstract}

(C) 2007 Elsevier B.V. All rights reserved.

Keywords: Phase I; Frozen; Dry ice; Distribution

\section{Introduction}

Clinical trial pharmaceuticals often require low temperature storage to achieve sufficient stability for an adequate shelf life. Therefore, frozen stock distribution within Phase I and II clinical trial networks is relatively common. Indeed, within the authors' experience at the Cancer Research UK Formulation Unit (a Phase I production unit), an internal data audit of clinical trial shipments over 12 months revealed that $23 \%$ of all clinical product transits had a frozen shipment requirement.

To maintain product quality, ensure patient safety and treatment efficacy, it is imperative that the distribution of frozen materials is suitably considered and appropriately handled. In recently published MHRA data (Taylor et al., 2006), "cold chain transportation" was listed at 7 in the top 10 trended deficiencies from UK Good Distribution Practice (GDP, 2007)

\footnotetext{
* Corresponding author. Tel.: +44 141548 2454; fax: +44 1415484903

E-mail address: moira.elliott@strath.ac.uk (M.A. Elliott).
}

inspections in 2004-2005. This demonstrates both regulatory interest and the importance of this aspect of pharmaceutical operations.

'Dry ice' is often chosen for frozen transits. The material's common nomenclature is derived from the ability to sublime to carbon dioxide gas. Thus, the internal parcel environment does not become 'wet,' a factor for consideration in pharmaceutical transport. Exceptionally low temperatures can be achieved during the transit with a sublimation temperature of $-78.5^{\circ} \mathrm{C}$ (Lide, 2006). Dry ice would be expected to maintain frozen conditions for as long as it persists, a time course influenced by the amount used, transit duration and external temperature (or heat stress) experienced during the shipment. These represent measurable test parameters that would contribute to a transit verification study.

The aim of this study was to firstly determine, and then to repeatedly test, appropriate transit arrangements for frozen clinical trial products using dry ice. These tests would be used to determine robust and reproducible frozen shipment arrangements to be operated by our Phase I unit. 


\section{Materials and methods}

\subsection{Transit container}

An expanded polystyrene transit container enclosed in white cardboard (Product code CL87-30 from Cool Logistics, Soulbury, UK) was used in all studies. External dimensions were $45 \mathrm{~cm} \times 45 \mathrm{~cm} \times 43 \mathrm{~cm}$, with internal dimensions $32 \mathrm{~cm} \times 32 \mathrm{~cm} \times 29.5 \mathrm{~cm}$.

\subsection{Dry ice}

Single blocks of dry ice (BOC, Manchester, UK) were obtained from the University of Strathclyde Chemistry Stores. Dry ice weight was determined before and after each test using a 34-kg top pan balance (Model LA34000 from Sartorius, Epsom, UK).

\subsection{External temperature logging devices}

External temperature was monitored at 30-min intervals using a single Sergeant Temp Version 5 temperature logger (Wessex Power Technology, Poole, UK). The temperature logger was bubble wrapped for transit protection and placed in a moulded recess in the exterior face of the transit container lid. At test conclusions, logging was stopped by data download from the device. Data were graphed and analysed using the logging device software program. Measurement capability is in the range -40 to $+70^{\circ} \mathrm{C}$ with accuracy to $\pm 0.5^{\circ} \mathrm{C}$. The supplier company provides annual re-calibration and certification.

\subsection{Internal temperature logging devices}

Internal parcel temperature was monitored at 34-s intervals using Marathon Products edl- $\mathrm{CO}_{2}$ ultra low temperature loggers (Wessex Power Technology, Poole, UK). At test conclusions, logging was stopped by data download from the device. Data were graphed and analysed using the logging device software program. Measurement capability is in the range -81 to $+30^{\circ} \mathrm{C}$ with accuracy to $\pm 0.2^{\circ} \mathrm{C}$ at $0^{\circ} \mathrm{C}$, and $\pm 2.5^{\circ} \mathrm{C}$ at extremes. The supplier company provides annual re-calibration and certification.

\subsection{Dummy product packs}

Eight $132 \mathrm{~mm} \times 132 \mathrm{~mm} \times 50 \mathrm{~mm}$ white cardboard vial boxes with removable lids were used as dummy product packs (PakGen, Sevenoaks, UK). Three boxes of the eight carried an edl- $\mathrm{CO}_{2}$ temperature logging device with their internal card dividers removed to allow inclusion of the logger. All boxes used were empty of vial product with the intent of monitoring the surrounding environment that vials would experience, and not of the internal environment of individual vials.

For laboratory testing only, three dummy pack boxes were stacked three high in what was described as a 'tower' box arrangement. Each box had a temperature logger enclosed. The

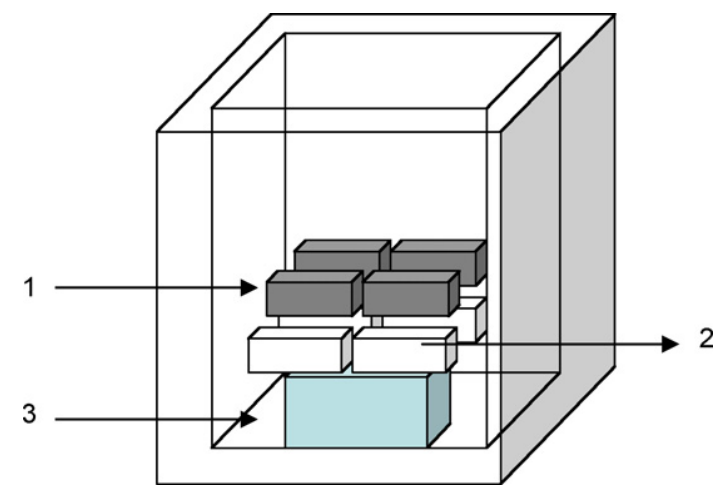

Fig. 1. 'Flat' box packing arrangement in the standard insulated transit container. Position 1 is the top box layer (with one edl- $\mathrm{CO}_{2}$ logger), position 2 the lower box layer (with two edl- $\mathrm{CO}_{2}$ loggers) and position 3 is the dry ice block. Box layers were bagged and bubble wrapped for transit protection.

tower was bubble wrapped and then placed in the transit container.

For some laboratory and all transit tests, eight dummy pack boxes were packed in two layers with four boxes in each, stacked two high in what was described as a 'flat' box arrangement (Fig. 1). The base box layer had a temperature logger in opposite corners of the square created by the four box arrangement (loggers 2 and 3). The top layer had a temperature logger in one of the four corners placed directly over a lower layer logger position (logger 1). Before placement into the transit container, each layer of boxes was first wrapped in a grip seal bag (four boxes per bag), then outer bubble wrapped as per Unit protocol for protection during transit. Polystyrene chips took up the remaining space to reduce excessive movement of the contents during transit.

\subsection{Laboratory tests}

Laboratory tests firstly determined the minimum temperature achieved by placing edl- $\mathrm{CO}_{2}$ temperature loggers in direct contact with the dry ice block on the base of a transit container. Further laboratory tests determined the temperatures recorded by loggers placed within dummy packs in both 'tower' and 'flat' box packaging arrangements.

\subsection{Shipping tests}

The transit container was packaged with dry ice, dummy packs in a 'flat' box arrangement and temperature loggers as previously described. The whole transit container was then brown paper wrapped and red labeled to identify a temperature trial in progress and to warn the recipient not to open the package. Transits were made in standard, non-refrigerated vehicles using a Glasgow to London return route.

\section{Results}

Laboratory tests recorded temperatures of $-61.4{ }^{\circ} \mathrm{C}$ when loggers were placed in direct contact with the ice block. Introduction of loggers within dummy product packs produced some 
Table 1

Results from internal parcel temperature monitoring for laboratory based tests using different packaging configurations

\begin{tabular}{|c|c|c|c|}
\hline & $\begin{array}{l}\text { Direct contact } \\
\text { with dry ice }\left({ }^{\circ} \mathrm{C}\right)\end{array}$ & $\begin{array}{l}\text { Tower box } \\
\text { arrangement }\left({ }^{\circ} \mathrm{C}\right)\end{array}$ & $\begin{array}{l}\text { Flat box } \\
\text { arrangement }\left({ }^{\circ} \mathrm{C}\right)\end{array}$ \\
\hline Logger $1^{\mathrm{a}}$ & -53.6 & $-10.1^{b}$ & $-21.9^{c}$ \\
\hline Logger $2^{\mathrm{a}}$ & -57.8 & $-24.6^{\mathrm{c}}$ & $-48.9^{\mathrm{d}}$ \\
\hline Logger $3^{a}$ & -61.4 & $-44.7^{\mathrm{d}}$ & $-42.2^{\mathrm{d}}$ \\
\hline Dry ice weight at study start & $8.795 \mathrm{~kg}$ & $8.723 \mathrm{~kg}$ & $9.601 \mathrm{~kg}$ \\
\hline
\end{tabular}

One block of dry ice was used in each test.

a edl- $\mathrm{CO}_{2}$ ultra low temperature data logger. Temperatures noted are average over the period of study.

b Top position.

c Middle position.

d Base position (all with respect to the parcel base).

Table 2

Results from internal parcel temperature monitoring for transit tests using the 'flat' box packaging configuration

\begin{tabular}{|c|c|c|c|c|c|}
\hline & Test 1 in ${ }^{\circ} \mathrm{C}$ (avg./max.) & Test 2 in ${ }^{\circ} \mathrm{C}$ (avg./max.) & Test 3 in ${ }^{\circ} \mathrm{C}$ (avg./max.) & Test 4 in ${ }^{\circ} \mathrm{C}$ (avg./max.) & Test 5 in ${ }^{\circ} \mathrm{C}$ (avg./max.) \\
\hline Logger $1^{\mathrm{a}, \mathrm{b}}$ & $-30.2 /-20.3$ & $-20.7 /-12.2$ & $-23.3 /-11.3$ & $-21.5 /-17.2$ & $-13.6 /-10.0$ \\
\hline Logger $2^{\mathrm{a}, \mathrm{c}}$ & $-51.9 /-41.8$ & $-34.8 /-10.7$ & $-41.4 /-29.4$ & $-44.6 /-31.7$ & $-36.5 /-30.42$ \\
\hline $\operatorname{Logger} 3^{\mathrm{a}, \mathrm{c}}$ & $-50.4 /-42.8$ & $-41.8 /-23.3$ & $-38.5 /-24.7$ & $-47.6 /-33.8$ & $-38.9 /-33.6$ \\
\hline Exterior $^{\mathrm{d}}$ & +11.4 & +13.2 & +10.9 & +20.1 & +13.4 \\
\hline Dry ice at study start & $7.864 \mathrm{~kg}$ & $8.482 \mathrm{~kg}$ & $9.319 \mathrm{~kg}$ & $8.358 \mathrm{~kg}$ & $8.174 \mathrm{~kg}$ \\
\hline
\end{tabular}

One block of dry ice was used in each test.

a edl- $\mathrm{CO}_{2}$ ultra low temperature logger.

b Top layer.

c Base layer (with respect to the parcel base).

d Sergeant Temp temperature logger (mean kinetic temperature is presented).

insulating effects, with the recorded value rising to $-48.9^{\circ} \mathrm{C}$. Not surprisingly, box positioning had an effect. Increased distance from the dry ice block increased the average recorded temperatures in the 'tower' box arrangement. It was concluded that 'flat' box packing would be used in real time transits, as the maximum average temperatures were lower than in the stacked 'tower' box arrangement (Table 1, -48.9 to $-21.9^{\circ} \mathrm{C}$ in the former case, -44.7 to $-10.1{ }^{\circ} \mathrm{C}$ in the latter).

Transit tests were all successfully completed and were compliant with the nominal specification of individual internal temperatures not exceeding a maximum $-10{ }^{\circ} \mathrm{C}$ during transport (Table 2 and Fig. 2). "Dips" in temperature were routinely observed co-incident in Fig. 2 with very early morning (approximately 03.00-07.00) or late evening (approximately 21.30-02.00), suggested times of minimum parcel handling or road transit disturbance.

The single block of dry ice used in each test had weight in the range $7.864-9.319 \mathrm{~kg}$. In all transit tests, not more than $5 \mathrm{~kg}$ of the ice had been consumed during a maximum of $52.5 \mathrm{~h}$ in transit (Table 3).

The 'stress' exerted on the parcel was recorded by external parcel temperature monitoring. To allow interpretation of normal fluctuating day and night temperatures, the mean kinetic external temperature of each transit was calculated. All transit tests were carried out in summer to provide maximum external seasonal temperature. Interestingly, during these summer tests one transit in particular (Test 4 in Table 2) was sent out during a period of un-seasonally high temperatures recorded in Glasgow and London with readings regularly in the high $20^{\circ} \mathrm{C}$ range for Glasgow, and in excess of $30^{\circ} \mathrm{C}$ for London (July 2006). Although mean kinetic temperatures ranged from +10.9 to $+20.1{ }^{\circ} \mathrm{C}$, dry ice sublimation rate (approximated from the used dry ice weight divided by transit time) was relatively constant ranging from 0.076 to $0.096 \mathrm{~kg} / \mathrm{h}$, equivalent to an overall average rate of $0.085 \mathrm{~kg} / \mathrm{h}( \pm 0.007 \mathrm{~kg} / \mathrm{h})$.

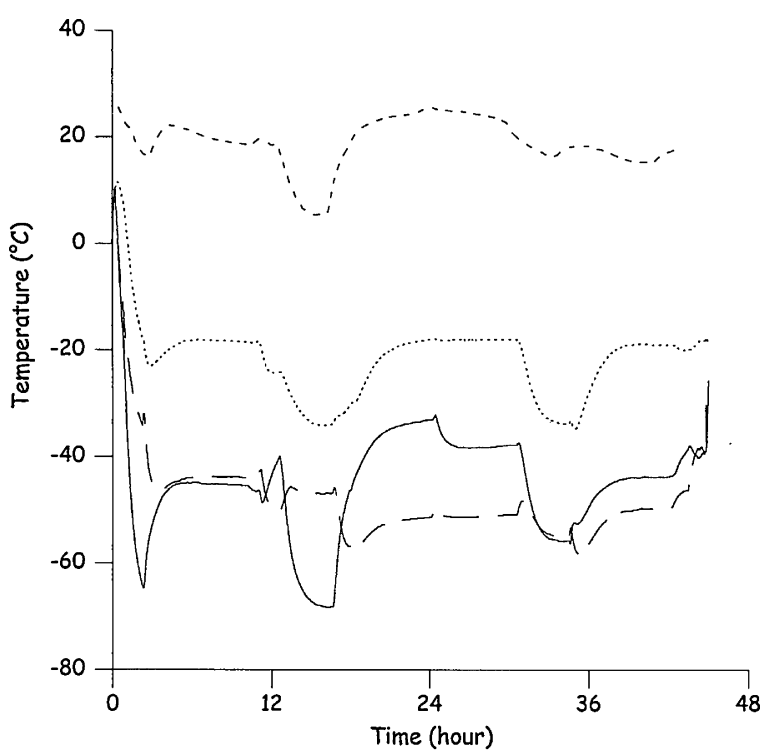

Fig. 2. Test 4 temperature data covering a Glasgow to London return transit with external mean kinetic temperature of $+20.1^{\circ} \mathrm{C}$. External temperature data are shown as -- -, internal temperature data for an upper layer box as $\cdots$, internal temperature data for two lower layer boxes as _ — — and —_. Forty-eight hours of data are shown, starting at $14.30 \mathrm{~h}$. 
Table 3

Approximate dry ice sublimation rates for the parcels under test (the test numbers correlate to Table 2 results)

\begin{tabular}{|c|c|c|c|c|c|}
\hline & Test 1 & Test 2 & Test 3 & Test 4 & Test 5 \\
\hline Dry ice used during transit & $4.256 \mathrm{~kg}$ & $4.519 \mathrm{~kg}$ & $4.353 \mathrm{~kg}$ & $4.725 \mathrm{~kg}$ & $3.983 \mathrm{~kg}$ \\
\hline Total duration of transit test & $51 \mathrm{~h}$ & $52 \mathrm{~h}$ & $51 \mathrm{~h}$ & $49 \mathrm{~h}$ & $52.5 \mathrm{~h}$ \\
\hline Approx. rate of sublimation & $0.083 \mathrm{~kg} / \mathrm{h}$ & $0.087 \mathrm{~kg} / \mathrm{h}$ & $0.085 \mathrm{~kg} / \mathrm{h}$ & $0.096 \mathrm{~kg} / \mathrm{h}$ & $0.076 \mathrm{~kg} / \mathrm{h}$ \\
\hline
\end{tabular}

\section{Discussion}

We have previously reported on the importance of cold chain product management (Elliott and Halbert, 2005) where transit conditions that can affect product stability and therefore efficacy and patient safety, or have financial penalty if a product is rejected, were all discussed. In frozen product transport, the patient safety risks are certainly equivalent, if not higher, as frozen materials too will potentially suffer detriment to product quality following any significant out of specification temperature excursions.

The complexity of the verification studies needed for assessment of shipping conditions may be linked to both the type of product and to the distribution network in which it is destined to travel. Global distribution with multipart and intricate networks undoubtedly will attract more difficulties than a straight 'A to B' overnight land based delivery. Nonetheless, the aim is the same as summarized by Ennis, 2006, in that the clinical trial product received by a pharmacy for patient dosing should be exactly of the same quality as that certified by the manufacturer's Qualified Person $(\mathrm{QP})$.

In this study, we sought straightforward, robust and repeatable frozen shipping conditions for UK based delivery that would be within the operational capabilities of a small Phase I clinical trial distribution network. The system chosen, often described as 'passive' as there is a simple reliance on dry ice for deep frozen internal parcel conditions, has been shown to satisfy all of these aims. Internal parcel temperatures did not rise above the specification of not greater than $-10{ }^{\circ} \mathrm{C}$, and indeed on average were often much lower. Conditions were maintained for the duration of an overnight delivery and beyond, in fact up to $52.5 \mathrm{~h}$ providing a significant time contingency for stray deliveries. Repeatability was shown in five individual real time transit tests, all of which were stressed by monitored external summer temperatures.

In summary, the frozen transit conditions selected have shown satisfactory real time shipment performance in terms of robustness and repeatability, were straightforward in terms of operator training and were cost effective. More importantly, the system was able to provide temporary storage conditions that would ensure suitability for product use on arrival at the destination, a process that is designed to ultimately protect patients on clinical trials.

\section{Acknowledgements}

This work was funded by Cancer Research UK. The work of the Formulation Unit staff, and of the pre-clinical team in the London Drug Development Office is also gratefully acknowledged.

\section{References}

Elliott, M.A., Halbert, G.W., 2005. Maintaining the cold chain shipping environment for Phase I clinical trial distribution. Int. J. Pharm. 299, 49-54

Ennis, C., 2006. Control of cold chain distribution. Eur. J. Parent. Pharm. Sci. 11 (3), 67-69.

GDP, 2007. Guidelines on good distribution practice of medicinal products for human use (94/C63/03) (text with EEA relevance).

Lide, D.R., 2006. CRC Handbook of Chemistry and Physics, 87th ed. CRC Press.

Taylor, J., Heddell, G., Holloway, I., Matthews, G., Rees, I., 2006. GMP and GDP: a review of regulatory inspection findings and defective medicines reports for 2004-05. Pharm. J. 276, 593-596. 